International Journal of Pure and Applied Mathematics

Volume 105 No. 3 2015, 529-535

ISSN: 1311-8080 (printed version); ISSN: 1314-3395 (on-line version)

url: http://www.ijpam.eu

doi: http://dx.doi.org/10.12732/ijpam.v105i3.18

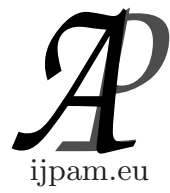

\title{
ANALYTICAL SOLUTIONS FOR LINEAR VOLTERRA AND ABEL INTEGRAL EQUATIONS OF SECOND KIND USING A POWER SERIES METHOD
}

\author{
Akimov Andrey $^{1 \S}$, Agafonova Alena ${ }^{2}$ \\ ${ }^{1,2}$ Bashkir State University Sterlitamak Branch \\ 453103, Lenina street 47A \\ Sterlitamak, RUSSIA
}

\begin{abstract}
Even though they have a rather specialized structure, Volterra and Abel integral equations form an important class of integral equations in applications. This happens because completely independent problems lead to the solution of such equations. In this paper we consider the liner Volterra and Abel integral equations of second kind. Authors have been proposed a new method for constructing solutions of Abel integral equations by a power series.
\end{abstract}

AMS Subject Classification: 45D05

Key Words: generalized Abel integral equations, integral equation, power series

\section{Introduction and Preliminary}

In this paper, we use the method of generalized power series, to solve linear Volterra integral equations of the second kind. This power series are undetermined coefficients method, or a method based on the application of the Taylor series. The result obtained in the form of generalized power series solution further converted to the inversion formula of the integral equation. One such method is the representation of the solution of the equation in the form of a power series [1], [2]. We also consider Abel equation of the second order [3] as

Received: October 27, 2015

(C) 2015 Academic Publications, Ltd.

$\S_{\text {Correspondence author }}$ url: www.acadpubl.eu 
a special case of the equation of Volterra.

Consider the nonlinear Volterra integral equation of second kind

$$
v(x)=g(x)+\int_{0}^{x} K(x, t) G[v(t)] d t, \quad x \in[0,1]
$$

where the kernel $G(x, t)$ and the function $f(x)$ are given real-valued functions, and $G(v(x, t))$ is a nonlinear function of $v(t)$ such as $u^{2}(x), \cos (u(x))$ and $e^{u(x)}$. Suppose the solution of equation (1) be as

$$
v(x)=a_{0}+a_{1} x,
$$

where $a_{0}=g(0)=v(0)$ and $c_{1}$ is a unknown parameter. By substituting Eq.(2) into Eq.(1) with simple calculations, we get

$$
\left(a_{1}-c_{1}\right) x+Q\left(x^{2}\right)=0,
$$

where $Q\left(x^{2}\right)$ is a polynomial of order greater than one. By neglecting $Q\left(x^{2}\right)$, we have linear equation of $a_{1}$ in the form,

$$
a_{1}=c_{1},
$$

where the parameter $a_{1}$ of $x$ in Eq. (2) is then obtained. In the next step, we assume that the solution of Eq.(1) is

$$
v(x)=a_{0}+a_{1} x+a_{2} x^{2},
$$

where $a_{0}$ and $a_{1}$ both are known and $a_{2}$ is unknown parameter. By substituting Eq.(5) into Eq.(1), we get

$$
\left(a_{2}-c_{2}\right) x^{2}+Q\left(x^{3}\right)=0,
$$

where $Q\left(x^{3}\right)$ is a polynomial of order greater than two. By neglecting $Q\left(x^{3}\right)$, we have linear equation of $a_{2}$ in the form,

$$
a_{2}=c_{2},
$$

the unknown parameter $a_{2}$ of $x^{2}$ in Eq.(5) is then obtained. Having repeated the above procedure for $n$ iterations, a power series of the following form is derived:

$$
v(x)=a_{0}+a_{1} x+a_{2} x^{2}+\ldots+a_{n} x^{n}
$$

Eq.(8) is an approximation for the exact solution $v(x)$ of Eq.(1) in the interval $[0,1]$. 


\section{Linear Volterra and Abel Integral Equations}

Consider the following linear Volterra integral equation [1]:

$$
v(x)=1-x-x^{2}+\int_{0}^{x}(x-t) v(t) d t
$$

$a_{0}=v(0)=f(0)=1$ as the initial condition, suppose the solution of Eq.(9) be

$$
v(x)=a_{0}+a_{1} x=1+a_{1} x
$$

Substitute Eq.(10) in Eq.(9) we get

$$
1+a_{1} x=1-x-x^{2}+\int_{0}^{x}(x-t) a_{1} t d t
$$

By integrating and solving we get $6 a_{1} x+6 x+3 x^{2}-a_{1} x^{3}=0$.

By neglecting $\left(3 x^{2}-a_{1} x^{3}\right)$, therefore $a_{1}=-1$. Substitute $a_{1}=-1$ in Eq.(10) we get $v(x)=1-x$.

Suppose the solution of Eq.(10) be as

$$
v(x)=a_{0}+a_{1} x+a_{2} x^{2}=1-x+a_{2} x^{2} .
$$

Substitute Eq.(11) in Eq.(9)we get

$$
1-x+a_{2} x^{2}=1-x-x^{2}+\int_{0}^{x}(x-t)\left(1-t+a_{2} t^{2}\right) d t .
$$

By integrating and solving we get $6\left(2 a_{2}+1\right) x^{2}-2 x^{3}-a_{2} x^{4}=0$.

By neglecting $a_{2} x^{4}$, therefore $2 a_{2}=-1$.

Substitute $a_{2}$ in Eq.(11) we get

$$
v(x)=1-x-\frac{1}{2} x^{2} .
$$

Continue by this way we will get series of the form

$$
v(x)=1-x-\frac{x^{2}}{2}-\frac{x^{3}}{6}+\ldots,
$$


so that gives the exact solution

$$
v(x)=2-e^{x}
$$

Let's now consider Abel integral equation of the second kind:

$$
y(x)-\int_{0}^{x} \frac{y(t) d t}{\sqrt{x-t}}=f(x) .
$$

Let's assume that the function $f(x)$ can be represented as follows:

$$
f(x)=c_{0}+c_{1}(x-a)+c_{2}(x-a)^{2}+\ldots+c_{n}(x-a)^{n}+\ldots
$$

The solution of this equation will be sought in the form of a sum of two generalized power series:

$$
y(x)=x^{\frac{1}{2}} \sum_{n=0}^{+\infty} a_{n} x^{n}+\sum_{n=0}^{+\infty} b_{n} x^{n}
$$

Substituting the power series (13), (14) into equation (12), performing the calculations, obtain

$$
\begin{gathered}
\sum_{n=0}^{+\infty} c_{n} x^{n}=x^{\frac{1}{2}} \sum_{n=0}^{+\infty} a_{n} x^{n}+\sum_{n=0}^{+\infty} b_{n} x^{n}-\int_{0}^{x} \frac{x^{\frac{1}{2}} \sum_{n=0}^{+\infty} a_{n} x^{n}+\sum_{n=0}^{+\infty} b_{n} x^{n}}{\sqrt{x-t}} d t= \\
=x^{\frac{1}{2}} \sum_{n=0}^{+\infty} a_{n} x^{n}+\sum_{n=0}^{+\infty} b_{n} x^{n}- \\
-\sum_{n=0}^{+\infty} a_{n} x^{n+1} \mathrm{~B}\left(\frac{1}{2}, n+\frac{3}{2}\right)-\sum_{n=0}^{+\infty} b_{n} x^{n+\frac{1}{2}} \mathrm{~B}\left(\frac{1}{2}, n+1\right)
\end{gathered}
$$

Then by equating the terms with identical powers of $x$ we get:

$$
\begin{gathered}
a_{m}=b_{m} \mathrm{~B}\left(\frac{1}{2}, m+1\right), b_{0}=c_{0}, \\
b_{m+1}=a_{m} \mathrm{~B}\left(\frac{1}{2}, m+\frac{3}{2}\right)+c_{m+1} \\
b_{m+1}=b_{m} \mathrm{~B}\left(\frac{1}{2}, m+1\right) \mathrm{B}\left(\frac{1}{2}, m+\frac{3}{2}\right)+c_{m+1},
\end{gathered}
$$




$$
\begin{aligned}
& \sum_{n=0}^{+\infty} b_{n} x^{n}=\sum_{p=0}^{+\infty} \sum_{k=0}^{p} c_{k} x^{p} \pi^{p-k} \frac{k !}{p !}= \\
& =\sum_{p=0}^{+\infty} \sum_{k=0}^{p} c_{k} x^{p} \frac{\pi^{p-k}}{(p-k-1) !} \cdot \frac{\Gamma(k+1) \Gamma(p-k)}{\Gamma(p+1)}= \\
& =\sum_{k=0}^{+\infty} c_{k} x^{k}+\sum_{k=0}^{+\infty} \sum_{n=0}^{+\infty} c_{k} x^{n+k+1} \frac{\pi^{n+1}}{n !} \mathrm{B}(k+1, n+1), \\
& x^{\frac{1}{2}} \sum_{n=0}^{+\infty} a_{n} x^{n}=x^{\frac{1}{2}} \sum_{p=0}^{+\infty} \sum_{k=0}^{p} c_{k} x^{p} k ! \pi^{p-k} \frac{2^{p+1}}{(2 p+1) ! !}= \\
& =x^{\frac{1}{2}} \sum_{p=0}^{+\infty} \sum_{k=0}^{p} c_{k} x^{p} \pi^{p-k} \frac{\Gamma\left(\frac{1}{2}\right) \Gamma(k+1)}{\Gamma\left(k+\frac{3}{2}\right)} \cdot \frac{1}{(p-k-1) !} \cdot \frac{\Gamma\left(k+\frac{3}{2}\right) \Gamma(p-k)}{\Gamma\left(p+\frac{3}{2}\right)}= \\
& =\sum_{k=0}^{+\infty} c_{k} x^{k+\frac{1}{2}} \mathrm{~B}\left(\frac{1}{2}, k+1\right)+ \\
& +\sum_{k=0}^{+\infty} \sum_{n=0}^{+\infty} c_{k} x^{n+k+\frac{3}{2}} \mathrm{~B}\left(\frac{3}{2}, k+1\right) \frac{\pi^{n+1}}{n !} \mathrm{B}\left(k+\frac{3}{2}, n+1\right)
\end{aligned}
$$

and this implies that our solution can be written as

$$
\begin{gathered}
y(x)=\sum_{k=0}^{+\infty} c_{k} x^{k+\frac{1}{2}} \mathrm{~B}\left(\frac{1}{2}, k+1\right)+\sum_{k=0}^{+\infty} \sum_{n=0}^{+\infty} c_{k} x^{n+k+1} \frac{\pi^{n+1}}{n !} \mathrm{B}(k+1, n+1)+ \\
+\sum_{k=0}^{+\infty} c_{k} x^{k}+\sum_{k=0}^{+\infty} \sum_{n=0}^{+\infty} c_{k} x^{n+k+\frac{3}{2}} \mathrm{~B}\left(\frac{3}{2}, k+1\right) \frac{\pi^{n+1}}{n !} \mathrm{B}\left(k+\frac{3}{2}, n+1\right), \\
F(x)=\sum_{k=0}^{+\infty} c_{k} x^{k}+\sum_{k=0}^{+\infty} c_{k} x^{k+\frac{1}{2}} \mathrm{~B}\left(\frac{1}{2}, k+1\right)= \\
=f(x)+\sum_{k=0}^{+\infty} x^{k+\frac{1}{2}} \int_{0}^{1} c_{k} t^{k}(1-t)^{-\frac{1}{2}} d t= \\
=f(x)+\int_{0}^{x} \frac{\sum_{k=0}^{+\infty} c_{k} t^{k}}{\sqrt{x-t}} d t=f(x)+\int_{0}^{x} \frac{f(t) d t}{\sqrt{x-t}}
\end{gathered}
$$




$$
\begin{gathered}
I=\sum_{k=0}^{+\infty} \sum_{n=0}^{+\infty} c_{k} x^{n+k+1} \frac{\pi^{n+1}}{n !} \mathrm{B}(k+1, n+1)+ \\
+\sum_{k=0}^{+\infty} \sum_{n=0}^{+\infty} c_{k} x^{n+k+\frac{3}{2}} \mathrm{~B}\left(\frac{3}{2}, k+1\right) \frac{\pi^{n+1}}{n !} \mathrm{B}\left(k+\frac{3}{2}, n+1\right) \\
=\int_{0}^{1} \sum_{k=0}^{+\infty} \sum_{n=0}^{+\infty} c_{k} x^{n+k+1} \frac{\pi^{n+1} t^{k}(1-t)^{n}}{n !} d t+ \\
+\int_{0}^{x} \sum_{n=0}^{x+\infty} \frac{\pi^{n+1}(x-t)^{n}}{n !} \sum_{k=0}^{+\infty} c_{k} t^{k+\frac{1}{2}} \mathrm{~B}\left(\frac{3}{2}, k+1\right) d t= \\
\sum_{n=0}^{x} \frac{\pi^{n+1}(x-t)^{n}}{n !} \sum_{k=0}^{+\infty} c_{k} t^{k} d t+\pi \int_{0}^{x} e^{\pi(x-t)} \sum_{k=0}^{+\infty} c_{k} t^{k+\frac{1}{2}} \mathrm{~B}\left(\frac{3}{2}, k+1\right) d t= \\
=\int_{0}^{x} e^{\pi(x-t)}[f(t)+F(t)-f(t)] d t=\pi \int_{0}^{x(x-t)} F(t) d t
\end{gathered}
$$

And as a final result:

$$
y(x)=f(x)+\int_{0}^{x} \frac{f(t) d t}{\sqrt{x-t}}+\pi \int_{0}^{x} e^{\pi(x-t)} F(t) d t .
$$

This solution identical to the solution obtained in [5].

Theorem 1. If $f(t) \in C[a, b]$, then there exist a unique solution of equation Abel of second kind, which is expressed in the form (14).

The theorem is proved by direct verification that the formula (8) is a solution of equation (6).

\section{Conclusions}

In this paper, solution is obtained by power series method. This may be used in more combinatorial way to obtain solution of higher degree non-linear integral equations. 


\section{References}

[1] M. A. AL-Jawary , H. R. AL-Qaissy, Analytical approximate solutions for linear and nonlinear Volterra integral and integro- differential equations and some applications for the Lane-Emden equations using a power series method Mathematical theory and Modeling, 4, No. 8, (2014), 133-152.

[2] Tahmasbi, O. S. Fard, Numerical solution of linear Volterra integral equations system of the second kind, Applied Mathematics and Computation, 3, No. 2,(2008), 097-103. doi:10.1016/j.amc.2007.12.041

[3] Akimov A.A., On uniqueness Morawetz problem for the Chaplygin equation, International Journal of Pure and Applied Mathematics, 97, No. 3, (2014), 369-375. doi:10.12732/ijpam.v97i3.9

[4] A. Akimov, G. Galiaskarova The solution of the Darboux problem for the telegraph equation with deviation from the characteristic, International Journal of Pure and Applied Mathematics, 103, No. 2, (2015), 377-383. doi:10.12732/ijpam.v103i2.20

[5] A.V.Manzhirov and A.D.Polyanin, Handbook of Integral Equations: Solution Methods [in Russian], Factorial Press, Moscow, (2000). 
